\title{
Radial distribution of RNA genome packaged inside spherical viruses
}

\author{
Se Il Lee and T. T. Nguyen \\ Georgia Institute of Technology, School of Physics, 837 State Street, Atlanta, Georgia 30332-0430
}

(Dated: October 24, 2018)

\begin{abstract}
The problem of RNA genomes packaged inside spherical viruses is studied. The viral capsid is modeled as a hollowed sphere. The attraction between RNA molecules and the inner viral capsid is assumed to be non-specific and occurs at the inner capsid surface only. For small capsid attraction, it is found that monomer concentration of RNA molecules is maximum at the center of the capsid to maximize their configurational entropy. For stronger capsid attraction, RNA concentration peaks at some distance near the capsid. In the latter case, the competition between the branching of RNA secondary struture and its adsorption to the inner capsid results in the formation of a dense layer of RNA near capsid surface. The layer thickness is a slowly varying (logarithmic) function of the capsid inner radius. Consequently, for immediate strength of RNA-capsid interaction, the amount of RNA packaged inside a virus is proportional to the capsid area (or the number of proteins) instead of its volume. The numerical profiles describe reasonably well the experimentally observed RNA nucleotide concentration profiles of various viruses.

PACS numbers: 81.16.Dn, 87.16.A-, 87.19.rm
\end{abstract}

Viruses attract broad interests from physics community due to their ability of spontaneous self assembly. Many viruses can be produced both in-vivo and in-vitro as highly robust and monodisperse particles. As a result, beside biomedical applications, understanding virus assembly can also have novel promising applications in nanofabrication. At the basic level, viruses consist of viral genomes (RNA or DNA molecules) packaged inside a protective protein shell (viral capsid). The structures of viral capsids for most viruses are well understood from high-resolution experiments using cryoelectron microscopy or X-ray analysis [1, 2], as well as theoretical studies [3, 4]. Single-stranded RNA (ssRNA) viruses also package their genome spontanously during assembly. Several theoretical studies have demonstrated that the interaction between capsid proteins and RNA nucleotide basis plays an important role in the RNA packaging process, both energetically and kinetically [5, 6, 7, 8, 9]. However, unlike the structural study of viral capsid, there is still a lack of general understanding of struture of packaged RNA. In references 6, 8, ,9, different models of RNA packaging inside viruses were studied. However, all these works treat RNA molecules as linear flexible polymers. In this letter, we want to address the question of how RNA molecules are arranged inside a spherical virus, explicitly taking into acount the branching degree of freedom of RNA secondary structure.

We focus on a particular class of ssRNA viruses where the interaction between capsid proteins and RNA molecules is non-specific and occurs dominantly at the inner surface of the capsid. This is the case for viruses where basic amino acids are located on the surface and electrostatic interaction is strongly screened in the bulk solution (examples of such viruses are bacteriophage $\mathrm{MS}_{2}$, Q Beta, Dengue, Immature Yellow Fever,... generally viruses belonging to group $\mathrm{B}$ and $\mathrm{C}$ mentioned in Ref. 9). (In some viruses such as pariacoto virus [10], the viral capsid forces some fraction of RNA molecules to adopt it dodecahedron structure. In that case, the theory presented below should be applied to the free fraction of these RNAs.) Even though RNA-capsid interaction only occurs at the surface, RNA radial concentration profiles and the amount of RNA packaged inside a virus can be dictated by the strength of this interaction. The main result of this papers is that there are two different profiles for the radial RNA nucleotide concentration. For small capsid attraction, the RNA concentration is maximum at the center of the capsid. A representative virus (the Dengue virus) for this profile is shown in Fig. 1a. For larger capsid attraction, the RNA concentration is maximum at a distance close to (but always smaller than) the inner capsid radius. A representative virus (the bacteriophage $\mathrm{MS}_{2}$ ) for this profile is shown in Fig. 1b. For the later case, the RNA molecules form a dense layer at the inner capsid surface. The thickness of this layer varies very slowly (logarithmic) with the capsid radius. As a result, the amount of RNA packaged inside such viruses is proportional to the capsid area (or the number of capsid proteins) instead of its volume.

It is well known that ssRNA molecules fold on themselves due to base-pairing interaction between their nucleotides. Because nucleotide sequence of ssRNA molecules is not perfect for such pairing, their secondary structure is highly nonlinear. To the first approximation, RNA molecules are considered to be highly flexible branch polymers which can fluctuate freely over all possible branching configurations. Different branching configurations are described in the schematic way shown in Fig. 2, characterized by fugacities for "bi-functional" units (linear sequences), "tri-functional units" (branching points) and "endpoints" (stem-loops or hair-pins). We assume good solvent condition with repulsive interactions between the different units (with no "tertiary" pairing). Using a mean-field approximation [14] to a field the- 


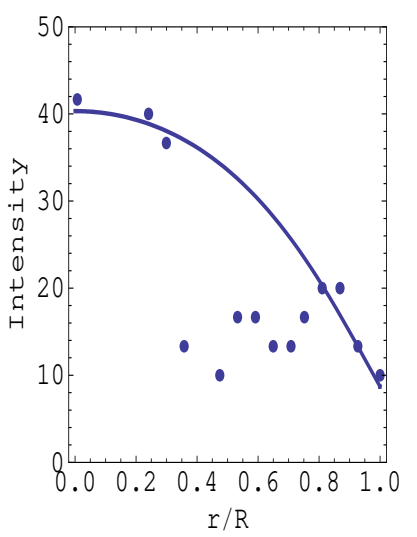

a)

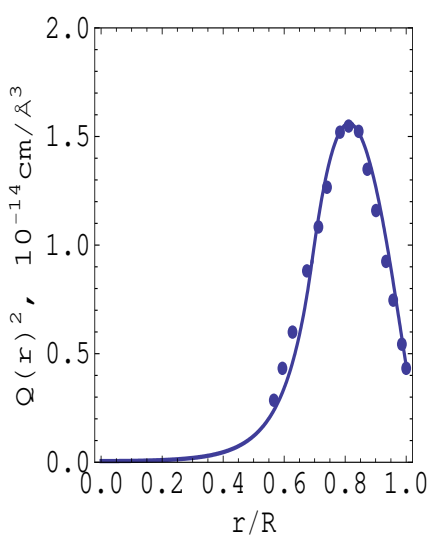

b)
FIG. 1: Two different profiles for RNA monomer concentration inside spherical viruses. Points are experimental data and solid lines are theoretical fit. a) Profile II, Eq. (9), fitted to RNA concentration of Dengue virus obtained from cryoelectron microscopy experiment [11]. b) Profile III, Eq. (11), fitted to RNA concentration of bacteriophage $\mathrm{MS}_{2}$ obtained from small angle neutron scattering experiment [12].

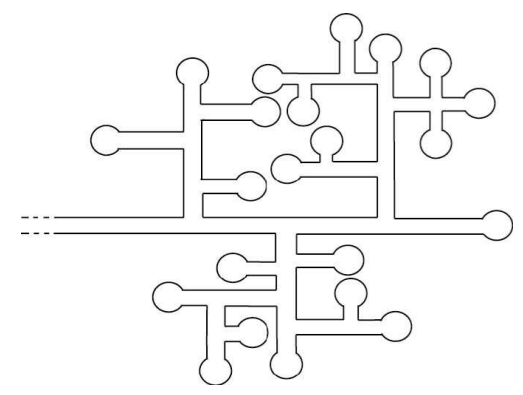

FIG. 2: Schematic representation of the secondary structure of a single-stranded RNA molecule as a collection of linear sections, branch-points, and end-points. The molecule can freely fluctuate between different branching configurations.

ory for solutions of branching polymers of this type [13], one can write down an expression for the free energy density of RNA solution $W[Q(\vec{r})]$ as

$$
\frac{W[Q(\vec{r})]}{m}=\frac{\epsilon}{2} Q(\vec{r})^{2}-\frac{w}{6} Q(\vec{r})^{3}+m u Q(\vec{r})^{4}-h Q(\vec{r}),
$$

where $\epsilon, w, h$ and $m$ are the fugacity of the monomers, branch points, the end-points and the whole polymers respectively. The coefficient $u$ is proportional to the second-order virial coefficient for monomer-monomer interaction (since RNA molecules are assumed to be in good solvent, $u$ is positive). $Q(\vec{r})$ is the order parameter of the field theory and is proportional to the concentration of end-points. Note that if one sets $w=0$ (the branching degree of freedom is suppressed), Eq. (11) recovers the well known expression for the free energy density of a solution of linear polymers [15]. Based on this mean-field expression, it is suggested that RNA are prone to a surface condensation which is different from that of linear polymer [14]. In this paper, we will use the mean-field expression, Eq. (1), to study how RNA molecules are packaged inside a virus. For simplicity, we model the viral capsid as a hollow sphere with inner radius $R$. We also assume that RNA molecules are radially distributed inside the capsid so that $Q(\vec{r}) \equiv Q(r)$ where $r$ is the radial distance from the center of viral capsid. As a result, the excess free energy of the RNA molecules packaged inside a capsid can be written as

$$
H_{\mathrm{MF}}=H_{s}+\int_{0}^{R} 4 \pi r^{2} d r\left\{\frac{m}{2}\left(\frac{d Q}{d r}\right)^{2}+\Delta W\right\},
$$

with $\Delta W[Q(r)]=W[Q(r)]-W\left[Q_{b u l k}\right]$. The first term in Eq. (2) denotes the interaction energy of the capsid proteins with the RNA molecules. Assuming this interaction occurs only at the inner capsid surface, $H_{s}$ can be written as the sum of contributions from monomers and endpoint adsorptions:

$$
H_{s}=4 \pi R^{2} m\left[-\gamma_{1} Q(R)-\gamma_{2} Q(R)^{2} / 2\right],
$$

where $\gamma_{1,2}$ are the strengths of the adsorption.

Due to the cubic term proportional to $w$ in Eq. (1), for small positive $\epsilon$, the free energy density $W(Q)$ has two minima, $Q_{D}$ and $Q_{C}$, corresponding to, respectively, the mean-field order parameter of a dilute bulk RNA solution and that of a condensed bulk RNA solution. A first-order condensation transition takes place when $W\left(Q_{D}\right)=W\left(Q_{C}\right)$. We will always assume RNA solution lies at this coexistence regime so that both the dilute and dense phases of RNA solution are close in energy. Therefore, we set bulk value $Q_{b u l k}=Q_{D}$. The equilibrium RNA concentration profile corresponds to the profile $Q(r)$ that minimizes the Hamiltionian Eq. (2). Setting the functional derivative, $\delta H_{M F} / \delta Q$ to zero, we obtain the Euler-Lagrangian equation

$$
\frac{d^{2} Q}{d r^{2}}+\frac{2}{r} \frac{d Q}{d r}-\frac{1}{m} \frac{\partial \Delta W}{\partial Q}=0
$$

and a boundary condition at the inner capsid surface:

$$
\left.\frac{d Q}{d r}\right|_{r=R}=\frac{H_{s}{ }^{\prime}[Q(R)]}{4 \pi R^{2} m}=-\gamma_{1}-\gamma_{2} Q(R) .
$$

To proceed further, we approximate $\Delta W$ using the double parabolic potential form [17]:

$$
\Delta W(Q)=\left\{\begin{array}{l}
\frac{1}{2} m \lambda_{D}^{2}\left(Q-Q_{D}\right)^{2} \text { for } Q<Q_{m} \\
\frac{1}{2} m \lambda_{C}^{2}\left(Q-Q_{C}\right)^{2} \text { for } Q>Q_{m}
\end{array},\right.
$$

where $Q_{m}=\left(\lambda_{D} Q_{D}+\lambda_{C} Q_{C}\right) /\left(\lambda_{D}+\lambda_{C}\right)$ is the point where the two parabolas cross each other forming a cusp. The two coefficients $\lambda_{D}^{2}, \lambda_{C}^{2}$ are the stiffness of the free energy density of RNA solution near the two minima. They are proportional to the corresponding correlation lengths 
of the two phases. In general, this double parabolic potential form for the free energy density breaks down near the critical temperature where the first order transition becomes second order, or when the fugacity of branch points, $w$, goes to 0 (the branching degree of freedom is suppressed and RNA molecules behave as a linear polymer). However, it was shown [14] that the mean-field expression, Eq. (1), breaks down before this limit is approached. If one stays within the limit of mean-field theory, the double parabola approximation is a reasonable approximation. We will come back to its limitation in later discussion. With this approximate form of $\Delta W$, Eq. (4) becomes linear and easy to solve. The general solution is a linear combination of $\exp \left( \pm \lambda_{D, C} r\right) / r$. There are three possible concentration profiles for the RNA molecules.

Profile $I$. If for all $r, Q(r)<Q_{m}$, then the solution to the Euler equation is

$$
Q(r)=-C_{10} \sinh \left(\lambda_{D} r\right) /\left(\lambda_{D} r\right)+Q_{D}
$$

where

$$
C_{10}=\frac{\left(\gamma_{1}+\gamma_{2} Q_{D}\right) R}{\cosh \left(\lambda_{D} R\right)+\left(\gamma_{2} R-1\right) \sinh \left(\lambda_{D} R\right) /\left(\lambda_{D} R\right)} .
$$

Because the interaction of the RNA monomers with the viral capsid is attractive, $\gamma_{1,2}>0$, the coefficient $C_{10}$ is a positive quantity. According to Eq. (7), this means that for all $r$, the endpoint (and monomer) concentration in this profile is always smaller than the bulk value, $Q(r)<$ $Q_{D}=Q_{b u l k}$. This is a non physical situation. Therefore, we discard this solution from later consideration.

Profile II. The second possibility is the case that for all $r, Q>Q_{m}$. Accordingly, the solution is

$$
Q(r)=-C_{20} \sinh \left(\lambda_{C} r\right) /\left(\lambda_{C} r\right)+Q_{C},
$$

where

$$
C_{20}=\frac{\left(\gamma_{1}+\gamma_{2} Q_{C}\right) R}{\cosh \left(\lambda_{C} R\right)+\left(\gamma_{2} R-1\right) \sinh \left(\lambda_{C} R\right) /\left(\lambda_{C} R\right)} .
$$

This solution is a monotonously decreasing function of $r$ and the RNA concentration is maximum at the center of the capsid. Because of the requirement that $Q(R)$ must be greater than $Q_{m}$, this profile is possible only for very weak adsorption (in practice, $\lambda_{C} R \gg 1$, this requirement means $\left.\left(\gamma_{1} / Q_{C}+\gamma_{2}\right) / \lambda_{D}<1\right)$. As a result, RNA monomers want to concentrate at the center of the capsid to gain their configurational entropy (minimizing the gradient term in Eq. (2) ).

Profile III. The third possibility is that $Q(r)$ passes through $Q_{m}$ at some distant $r=r_{0}\left(0<r_{0}<R\right)$ such that $Q\left(r=r_{0}\right)=Q_{m}$. We can interpret $r_{0}$ as the boundary between the dilute and the condensed phases of RNA molecules inside the capsid. Requiring the density profile
$Q(r)$ and its derivative $Q^{\prime}(r)$ to be continuous at $r_{0}$, we get

$$
Q(r)=\left\{\begin{array}{l}
\left(Q_{0}-Q_{D}\right) \frac{\sinh \left(\lambda_{D} r\right)}{\lambda_{D} r}+Q_{D} \quad \text { for } r<r_{0} \\
C_{31} \frac{\exp \left(\lambda_{C} r\right)}{\lambda_{C} r}+C_{32} \frac{\exp \left(-\lambda_{C} r\right)}{\lambda_{C} r}+Q_{C} \text { for } r_{0}<r<R
\end{array}\right.
$$

where $Q_{0}=Q(0)$ and

$$
\begin{aligned}
C_{31}= & -\exp \left[-\left(\lambda_{C}+\lambda_{D}\right) r_{0}\right]\left(Q_{0}-Q_{D}\right)\left(\lambda_{C} / \lambda_{D}-1\right) / 4 \\
& +\exp \left[-\left(\lambda_{C}-\lambda_{D}\right) r_{0}\right]\left(Q_{0}-Q_{D}\right)\left(\lambda_{C} / \lambda_{D}+1\right) / 4 \\
& -\exp \left(-\lambda_{C} r_{0}\right)\left(Q_{C}-Q_{D}\right)\left(\lambda_{C} r_{0}+1\right) / 2, \\
C_{32}= & \exp \left[\left(\lambda_{C}+\lambda_{D}\right) r_{0}\right]\left(Q_{0}-Q_{D}\right)\left(\lambda_{C} / \lambda_{D}-1\right) / 4 \\
& -\exp \left[\left(\lambda_{C}-\lambda_{D}\right) r_{0}\right]\left(Q_{0}-Q_{D}\right)\left(\lambda_{C} / \lambda_{D}+1\right) / 4 \\
& -\exp \left(\lambda_{C} r_{0}\right)\left(Q_{C}-Q_{D}\right)\left(\lambda_{C} r_{0}-1\right) / 2 .
\end{aligned}
$$

$r_{0}$ and $Q_{0}$ are two unknowns in the solution above. They can be solved by matching the boundary condition, Eq. (5), and the condition $Q\left(r_{0}\right)=Q_{m}$. The later condition gives

$$
Q_{0}=Q_{D}+\left(Q_{C}-Q_{D}\right) \frac{\lambda_{C}}{\lambda_{C}+\lambda_{D}} \frac{\lambda_{D} r_{0}}{\sinh \left(\lambda_{D} r_{0}\right)} .
$$

Substituting Eq. (11) and (12) into the boundary condition Eq. (5), we arrive at the equation for $r_{0}$ :

$$
\begin{aligned}
& \left(1+\frac{\gamma_{2}}{\lambda_{C}}-\frac{1}{\lambda_{C} R}\right)\left[-\frac{\lambda_{D} r_{0} \exp \left(-\lambda_{D} r_{0}\right)}{\sinh \left(\lambda_{D} r_{0}\right)}+1+\frac{\lambda_{D}}{\lambda_{C}}\right] u^{2} \\
& -2 \lambda_{s} R u \\
& -\left(1-\frac{\gamma_{2}}{\lambda_{C}}+\frac{1}{\lambda_{C} R}\right)\left[\frac{\lambda_{D} r_{0} \exp \left(\lambda_{D} r_{0}\right)}{\sinh \left(\lambda_{D} r_{0}\right)}-1-\frac{\lambda_{D}}{\lambda_{C}}\right]=0,
\end{aligned}
$$

where $u=\exp \left[\lambda_{C}\left(R-r_{0}\right)\right]$. The parameter

$$
\lambda_{s}=\left(1+\lambda_{D} / \lambda_{C}\right)\left(\gamma_{1}+\gamma_{2} Q_{C}\right) /\left(Q_{C}-Q_{D}\right) \text {, }
$$

is proportional to the strength of RNA adsorption at the inner capsid surface and has dimension of inverse length. Obtaining an analytical solution for $r_{0}$ from Eq. (13) is a highly non-trivial task and numerical solution is generally needed. Nevertheless, we can understand important qualitative features of the RNA concentration profile by solving for $r_{0}$ in the limit of strong capsid RNA adsorption $\left(\lambda_{s} R \gg 1\right)$ and small correlation length of RNA concentrated phase $\left(\lambda_{C} R \gg 1\right)$. In this limit, the first two terms in Eq. (13) are the two most dominant ones. Balancing them, we get $u \simeq 2 \lambda_{s} R$, or

$$
r_{0} \simeq R-\lambda_{C}{ }^{-1} \ln \left(2 \lambda_{s} R\right)
$$

As we mentioned above, $r_{0}$ can be considered as the boundary between a dense RNA phase near the capsid and a dilute RNA phase at the capsid center. The quantity $d=R-r_{0}$, therefore, can be considered the thickness of this dense RNA layer. According Eq. (15), $d \propto \ln R$ 
which is parametrically smaller than the capsid radius, $R$ [19]. In other words, our RNA concentration profile shows a dense RNA layer condensed on the inner capsid with thickness which varies very slowly with its radius. Consequently, the amount of RNA packaged inside the virus is proportional to the capsid area (or the number of capsid proteins) instead of its volume. In recent works [6, 9], a similar dependence is observed when positively charged amino acids of capsid proteins are located in their long flexible peptide arms. In their works, the thickness of RNA molecules (treated as linear polymers) layer depends on the length of these arms. On the other hand, for the class of viruses we study in this paper where the basic amino acids are located at the inner capsid surface instead of peptide arms, the competition between the branching degree of freedom of the secondary structure of RNA molecules and the attraction of capsid proteins is responsible for the layer structure and the thickness scales as $\ln R$. Another interesting feature of RNA concentration profile III is the fact that it does not peak at the inner capsid radius $R$ but at some smaller radius. This is the direct consequence of the boundary condition, Eq. (5) which forces the RNA concentration to decrease in the vicinity of the capsid.

In Fig. 1, we plot examples of the two profiles, Eq. (9) and Eq. (11), fitted to the experimental data for two viruses, the Dengue virus and bacteriophage $\mathrm{MS}_{2}$. The data for the Dengue virus was obtained using cryoelectron microscopy [11]. The data for bacteriophage $\mathrm{MS}_{2}$ was obtained using small angle neutron scattering measurements [12]. Both viruses have most of their basic amino acids located on the surface of inner capsid, therefore our model capsid can be used. Both theoretical profiles show reasonable agreement with experiment results.

So far, when solving the Euler-Lagrange equation for RNA density profile, we assume $Q(r)$ crosses the value $Q_{m}$ at most one time. Certainly, there is a possibility that $Q(r)$ can cross $Q_{m}$ multiple times as $r$ increases from zero to $R$. This results in an oscilating RNA concentration profile. One could easily extend our calculation presented in this paper to such a case by adding more piecewise solution to the ansatz, Eq. (11), and requiring $Q(r)$ and its derivative to be continuous at the crossing points. Such extension could offer insights, for e.g., into the oscillating radial profile of RNA molecules packaged inside Turnip Yellow Mosaic Virus (TYMV) [12]. Nevertheless, these cases are relatively uncommon and the calculations would go beyond the scope of this letter. We will address these cases in more detail in future study.

Naturally, one wants to know which RNA concentration profile is the most thermodynamically stable. To answer this question, one needs to substitute these profiles (Eq. (9) and Eq. (11)) into the original expression for the capsid excess free energy, Eq. (2), and compare the resulting energies. This is a tedious task. Numer- ically, it is found that for small adsorption strength of viral capsid, the second profile would be thermodynamically stable and RNA concentration is maximum at the capsid center. For stronger surface adsorption, the third profile is lower in energy. In this case, RNA molecules form a dense layer at the capsid and the RNA concentration is maximum at a finite radius smaller than $R$.

It is known [14] that the mean-field theory, Eq. (2), breaks down when the critical point is approached and the first order transition between dilute and condensed phases of RNA solution becomes of second order. Once this happens, a physical picture similar to that of a solution of branched polymer with frozen branching arrangement emerges 16]. In this case, the RNA molecules become unscreened and non-overlapped. For viruses with several packaged RNA molecules, each of them would adsorb independently onto the capsid and the layer thickness of each molecule scales as square root of its molecular weight. Conversely, if such separation between constituent viral genomes is observed, it would signal the breakdown of mean-field theory.

In conclusion, in this paper we found two different nucleotide concentration profiles of viral RNA molecules packaged inside spherical viruses. The theory applies to a class of viruses where capsid-RNA interaction occurs at the capsid surface only. For small interaction strength, the RNA monomer concentration is maximized at the center of the capsid to maximize their configurational entropy. For higher interaction strength, RNA forms a dense layer near the capsid surface. The thickness of this layer is a slowly varying (logarithmic) function of the inner capsid radius. In this case, the amount of packaged RNA would be proportional to capsid area (or number of capsid proteins) instead of its volume. The profiles describe reasonably well the experimental profiles for various viruses.

We would like to thank R. Zhang, V. Belyi, B. Shklovskii, T. Witten and R. Bruinsma for helpful discussions. Initial work on this problem was done with Huaming Li. Nguyen also acknowledges the Junior Faculty support from the Georgia Institute of Technology.

[1] H. Tsuruta, V. S. Reddy, W. R. Wikoff, and J. E. Johnson, J. Mol. Biol. 284, 1439 (1998).

[2] J. Johnson and R. Rueckert, in Structural biology of viruses, edited by W. Chiu, R. M. Burnett, and R. L. Garcea (Oxford University Press, New York, 1997).

[3] J. Lidmar, L. Mirny, and D. R. Nelson, Phys. Rev. E 68, 051910 (2003).

[4] T. T. Nguyen, R. F. Bruinsma, and W. M. Gelbart, Phys. Rev. Lett. 96, 078102 (2006).

[5] T. Hu and B. I. Shklovskii, Phys. Rev. E 75, 051901 (2007).

[6] V. A. Belyi and M. Muthukumar, Proc. Nat. Acad. Sci. USA 103, 17174 (2006). 
[7] J. Rudnick and R. F. Bruinsma, Phys. Rev. Lett. 94, 038101 (2005).

[8] P. van der Schoot and R. F. Bruinsma, Phys. Rev. E 71, 061928 (2005).

[9] T. Hu, R. Zhang, and B. I. Shklovskii (2006), qbio/0610009.

[10] L. Tang, K. N. Johnson, L. A. Ball, T. Lin, M. Yeager, and J. E. Johnson, Nat. Struct. Biol. 8, 77 (2001).

[11] R. J. Kuhn, W. Zhang, M. G. Rossmann, S. V. Pletnev, J. Corver, E. Lenches, C. T. Jones, S. Mukhopadhyay, P. R. Chipman, E. G. Strauss, et al., Cell 108, 717 (2002).

[12] B. Jacrot, C. Chauvin, and J. Witz, Nature 266, 417 (1977).

[13] T. C. Lubensky and J. Isaacson, Phys. Rev. A 20, 2130 (1979).
[14] T. T. Nguyen and R. F. Bruinsma, Phys. Rev. Lett. 97, 108102 (2006).

[15] P.-G. de Gennes, Scaling concepts in Polymer physics (Cornell University Press, Ithaca and London, 1988).

[16] M. Daoud and J. F. Joanny, J. Phys. (Paris) 42, 1359 (1981).

[17] R. Holyst and A. Poniewierski, Phys. Rev. B 36, 5628 (1987).

[18] P. G. de Gennes, Rev. Mod. Phys. 57, 827 (1985).

[19] The $\ln R$ dependence of $d$ is also obtained for the wetting layer on the surface of a colloid [17]. This is to be expected because Eq. (1) can be mapped onto the Cahn theory of wetting transition [14, 18]. Here we show that the negative curvature of the inner viral capsid apparently does not significantly affect this logarithmic dependency. 